\title{
L'ARTE E LO STATO: DA RUSKIN A KEYNES
}

\author{
GIORGIO LUNGHINI (*)
}

SunTO. - Nelle società in cui predomina il modo di produzione capitalistico, scrive Marx nell'incipit del Capitale, la ricchezza delle società si presenta come una "immane raccolta di merci" - e l'arte stessa oggi è diventata una merce. L'unicità di una opera d'arte è inseparabile dal suo essere incorporata nel tessuto della tradizione e secondo Benjamin ciò che va perduto nell'epoca della sua riproducibilità tecnica è l'aura dell'opera d'arte. La tecnica della riproduzione distacca l'oggetto riprodotto dal dominio della tradizione: per la prima volta nella storia del mondo, la riproduzione meccanica emancipa l'opera d'arte della sua dipendenza parassitica dal rito.

$* * *$

ABSTRACT. - The wealth of those societies in which the capitalist mode of production prevails, writes Marx in first page of Capital, presents itself as "an immense accumulation of commodities" - and art itself is now a commodity. The uniqueness of a work of art is inseparable from its being embedded in the fabric of tradition: and that which withers in the age of mechanical reproduction according to W. Benjamin is the "aura" of the work of art. The technique of reproduction detaches the reproduced object from the domain of tradition: for the first time in world history, mechanical reproduction emancipates the work of art from its parasitical dependence on ritual.

Per cogliere le caratteristiche rilevanti del processo di produzione, circolazione e fruizione delle opere d'arte, anche ai fini di una politica economica dell'arte, quel processo va collocato nel suo contesto storico. L'economia in cui viviamo non è una economia di scambio o cooperativa, è una economia capitalistica; meglio: una "economia monetaria di produzione", una economia in cui le merci vengono pro-

(*) Istituto Lombardo Accademia di Scienze e Lettere, Milano. Accademia Nazionale dei Lincei, IUSS Pavia, Italia. E-mail: giorgio.lunghini@iusspavia.it 
dotte in vista di un profitto, non in vista della soddisfazione di bisogni. Nelle società in cui predomina il modo di produzione capitalistico, scrive Marx nell'incipit del Capitale, la ricchezza delle società si presenta come una "immane raccolta di merci": arte compresa.

E nell'epoca della sua riproducibilità tecnica, scrive Benjamin ${ }^{1}$, la riproduzione dell'opera d'arte le sottrae l'autenticità: divenuta oggetto di consumo, essa perde la sua caratteristica tradizionale, l'aura. Prima di essere oggetto estetico, l'arte era oggetto di culto e di devozione. Al valore cultuale si sostituisce quello rappresentativo, una considerazione estetica, profana, indipendente dall'originario contesto religioso: l'opera vive ora nello spazio museale uno spazio separato rispetto allo spettatore. L'età della riproducibilità tecnica e del consumo da parte delle masse costituisce una trasformazione delle premesse sociali e delle modalità di percezione dell'opera d'arte, premesse e modalità che dipendono da due fattori: lo sviluppo della tecnica e l'affermarsi della società di massa.

\section{J. RUSKIN: UN MANIFESTO NOSTALGICO}

Nei modi di produzione precapitalistici le opere d'arte hanno la forma del "consumo signorile", mentre nel capitalismo prendono la forma della speculazione finanziaria: dal Principe mecenate al Mercante (e infatti il mercante d'arte e il connoisseur sono figure che compaiono nell'Ottocento).

La rappresentazione più efficace, e nostalgica, di quel che era l'arte prima del capitalismo si deve a Ruskin. Ruskin è noto sopratutto come studioso di estetica e come storico dell'arte, e tale principalmente fu prima del 1860; poi volle essere principalmente economista e riformatore sociale. Economia politica dell'arte è la cerniera tra i due

1 W. Benjamin, Das Kunstwerk im Zeitalter seiner technischen Reproduzierbarkeit (1935), in W. Benjamin: Gesammelte Schriften. Trad. it. L'opera d'arte nell' epoca della sua riproducibilità tecnica. Arte e società di massa, Torino, Einaudi, 1966.

2 The Political Economy of Art (1857), in The Works of John Ruskin. Ripubblicato nel 1880 con il titolo A Joy for Ever (dall'oggi abusato verso di Keats: “ $A$ Thing of Beauty is a Joy for Ever"). Trad. it. Economia politica dell'arte, Torino, Bollati Boringhieri, 1991. 
momenti del suo pensiero. Tuttavia nella sua opera non vi è soluzione di continuità, poiché la base della sua economia politica è la sua estetica, e fondamenta di entrambe sono l'etica e la religione. Il mondo vagheggiato da Ruskin è quello che egli immagina sia stato il Medioevo:

Esiste un magnifico attributo del colore nel tardo XII, l'intero XIII e il primo XIV secolo: l'unione di un colore con un altro con reciproca interferenza. Questo è un principio magnifico perché è eterno e universale, non solo nell'arte, ma nella vita umana. È il grande principio della Fratellanza, ottenuto non per mezzo dell'uguaglianza o della somiglianza, ma per mezzo del dare e del ricevere; le anime che sono diverse, le nazioni che sono diverse, e le nature che sono diverse, sono legate in un tutto nobile, perché ciascuna riceve qualcosa dei doni e della gloria delle altre.

Così nella critica d'arte come nella critica della società Ruskin rovescia la prospettiva corrente e vede nel Rinascimento la fine dell'Età dell'oro, il principio degli aridi 'tempi presenti', la riduzione della società da organismo vivente e solidale a macchina efficiente ma inanimata, il sopravvento della logica sul sentimento mistico, il prevalere del valore di scambio sul valore d'uso, la divisione smithiana del lavoro che si fa lavoro diviso, l'istituzione del denaro come nesso sociale: «la forza della ghinea che porti in tasca dipende totalmente dalla mancanza della stessa nella tasca del tuo vicino». Per Ruskin l'errore, il grande errore, del Rinascimento consiste nel credere che la scienza e l'arte siano la stessa cosa, e che il progresso dell'una sia necessariamente il perfezionamento dell'altra:

In realtà, esse sono non soltanto cose diverse, ma così opposte che, in novantanove casi su cento, il progresso dell'una fa retrocedere l'altra. Il male disperato dell'intero sistema del Rinascimento è che ogni senso di misura vi fu dimenticato, che la scienza fu ritenuta l'unico bene e che non si esaminò mai se gli uomini ne siano vivificati o paralizzati.

L'architetto del Rinascimento non lavora per il volgo, lavora soltanto per gli uomini di accademia e di corte:

Nella nuova precisione e nella legge accurata della forma classica gli uomini percepirono qualcosa che si confaceva in modo particolare alla esaltazione dello Stato: i principi se ne compiacquero, e i cortigiani. Il gotico era amico di tutti i cuori, e era universale, come la natura: poteva 
fabbricare un tempio per la preghiera dei popoli, o abbassarsi alla scala a chiocciola di un povero. Ma questa era un'architettura che non si sarebbe abbassata, che non conosceva l'umiltà, né la pietà. Non avrebbe costruito coi materiali di cui poteva disporre il povero; non avrebbe avuto per tetto stoppie o assicelle o neri tronchi di quercia; né per pareti ruvida pietra o mattone; né avrebbe avuto piccole finestre aperte là dove fosse necessario; nè si sarebbe rannicchiata, dovunque riuscisse a trovar posto, agli angoli delle vie. Sarebbe stata invece di pietra di taglio; e avrebbe avute le sue finestre e le sue porte, e le sue scale e i suoi pilastri, in ordine signorile e dimensioni maestose; e avrebbe avuto ali e corridoi, sale e giardini, come se la terra intera fosse sua. E le rozze capanne dei montanari e le fantastiche strade dei borghesi affaticati, sarebbero scomparse dalla sua via, come specie inferiore.

\section{Per Ruskin l'età dell'oro è invece il suo Medioevo:}

L'aspetto più mirabile delle scuole gotiche di architettura è proprio questo: che accettano i risultati del lavoro delle menti più umili, e da frammenti tanto visibilmente imperfetti, fanno con indulgenza sorgere un tutto grandioso e inattaccabile.

E all'arte applica le stesse regole di vita, semplici ma fondamentali, che fissa per riconoscere "i prodotti e risultati di un lavoro sano e dignitoso": 1) Non incoraggiare la fabbricazione di articoli alla cui produzione non partecipa l'invenzione, a meno che non siano assolutamente necessari. 2) Non richiedere la precisione di rifinitura per se stessa, ma soltanto per scopi pratici o nobili. 3) Non incoraggiare imitazioni o copie di qualsivoglia genere, se non con l'intento di conservare la testimonianza di grandi opere.

\section{J.M. KEYNES: DALla NOSTALGIA AL PROGETTO}

Per tutta la sua vita, da Bloomsbury in poi, Keynes è stato attento al mondo dell'arte e parte da dove Ruskin era arrivato, trasformando la nostalgia in progetto. Eccone alcuni passi, tratti da Autosufficienza nazionale e da L'arte e lo Stato':

3 J.M. Keynes, National Self-Sufficiency, «The New Statesman and Nation», 
«Nel secolo XIX si sviluppò fino a un livello stravagante il criterio del tornaconto finanziario, come criterio per valutare l'opportunità di intraprendere una iniziativa di natura sia privata sia pubblica. Ogni manifestazione vitale fu trasformata in una sorta di parodia dell'incubo del contabile. Invece di utilizzare l'immenso incremento delle risorse materiali e tecniche per costruire la città delle meraviglie, si crearono $i$ bassifondi; e si pensò che fosse giusto e ragionevole farlo perche questi, secondo il criterio dell'impresa privata, "fruttavano", mentre la città delle meraviglie sarebbe stata, si pensava, un atto di follia che avrebbe - nell'imbecille linguaggio di stile finanziario - "ipotecato il futuro". Ma nessuno può credere oggi che l'edificazione di opere grandi e belle possa impoverire il futuro, a meno che non sia ossessionato da false analogie tratte da una astratta mentalita contabile.

La stessa regola autodistruttiva di calcolo finanziario governa ogni altro aspetto della vita. Distruggiamo le campagne perche le bellezze naturali non hanno valore economico. Probabilmente saremmo capaci di fermare il sole e le stelle perche non ci danno alcun dividendo. Se fossi oggi al potere cercherei subito di dotare le nostre principali città di tutto ciò che è connesso all'arte e alla civiltà al più alto livello raggiungibile da ciascun cittadino, convinto che sarei in grado di affrontare le spese di tutto ciò a cui darei vita e fiducioso che il denaro così speso non solo sarebbe piu utile di ogni sussidio di disoccupazione, ma renderebbe inutile tale sussidio.

II mondo antico era consapevole che il popolo aveva bisogno degli spettacoli circensi come del pane. A parte le esigenze politiche, i suoi governanti spendevano, per la propria gloria e soddisfazione, una quota rilevante della ricchezza nazionale per cerimonie, opere d'arte e grandiosi palazzi. Tali politiche, consuetudini e tradizioni non erano confinate al mondo Greco e Romano. Nei secoli diciottesimo e diciannovesimo la ricca nobiltà continuerà in modo privato, riservato e attutito quello che era stato il compito del monarca e dello Stato, con la Chiesa più o meno in declino. Ma nel diciottesimo secolo si fece strada una nuova visione delle funzioni dello Stato e della società, visione che raggiunse l'apice nel diciannovesimo secolo e che ancora oggi appare dominante.

Questa visione è rappresentata dall'ideale utilitarista e economico si potrebbe quasi dire finanziario - come l'unico rispettabile proposito

1933, ora in The Collected Writings. Trad. it. Autosufficienza nazionale, in J.M. Keynes, La fine del Laissez-faire e altri scritti, Torino, Bollati Boringhieri, 1991. J.M. Keynes, Art and the State, «The Listener», 1936, ora in CW. Trad.it.: http://host.uniroma3.it/facolta/economia/db/materiali/insegnamenti/646_4304.pdf 
della comunità nel suo complesso; la più orrenda eresia, forse, che abbia mai raggiunto l'orecchio di un popolo civile. I poeti e gli artisti hanno levato occasionalmente deboli voci contro quest'eresia. Credo che il Principe Consorte sia stato l'ultimo oppositore che si potesse trovare in alto loco, ma l'opinione del Tesoro ha prevalso. Non soltanto in pratica: la teoria è ugualmente potente. Ci siamo convinti che è assolutamente perverso per lo Stato spendere un centesimo per scopi non economici, e se sorge qualche occasione di spesa non economica che risulterebbe pubblicamente scandaloso perseguire, si pensa che basti capovolgere il cappello per chiedere l'elemosina ai privati.

Guardiamo alla conservazione dei monumenti nazionali ereditati dal passato come a una faccenda che debba dipendere dalle donazioni precarie e insufficienti di individui più attenti al bene pubblico di quanto non lo sia la stessa comunità. Dal momento che la Cattedrale di Lincoln, la quale incorona l'altura che per duemila anni è stata uno dei centri focali d'Inghilterra, potrebbe rovinare al suolo prima che il Tesoro prenda in considerazione un proposito troppo antieconomico da meritare un finanziamento pubblico, non c'è da meravigliarsi che le alte autorità non costruiscano più giardini pensili di Babilonia, non più piramidi, partenoni, colossei, cattedrali, palazzi, e neanche teatri lirici, palcoscenici, colonnati, boulevards e piazze pubbliche.

L'architettura è la più pubblica tra le arti, la meno privata nelle sue manifestazioni e la piu adatta a dare forma e corpo all'orgoglio civico e al senso di unità sociale. La musica viene subito dopo; poi le varie forme teatrali; quindi le arti plastiche e pittoriche - salvo che in alcuni aspetti della scultura e dell'arredamento, aspetti nei quali esse dovrebbero essere ausiliarie all'architettura; infine poesia e letteratura, per propria natura più private e personali.

Le nostre attuali politiche sono il riflesso di una certa filosofia politica, che io dico essere radicalmente sbagliata e che potrebbe persino, a lungo andare, minare la solidità delle nostre istituzioni. Cambieremo le nostre politiche soltanto se cambieremo la filosofia che vi è sottesa. Io ho indicato un punto di vista alternativo, e mi si lasci concludere con due esempi di ciò che potrebbe scaturire da un mutamento di mentalità - uno relativo alla conservazione di quanto abbiamo ereditato, l'altro in merito all'allargamento di quanto tramanderemo.

1. Si dovrebbe istituire una Commissione degli Immobili Pubblici con il potere di emettere ingiunzioni contro ogni atto di sfruttamento o sviluppo del terreno o ogni mutamento o demolizione di un edificio esistente, nel caso in cui essa ritenesse che un tale atto fosse contrario all'interesse generale, con il potere di erogare un compenso nella misura ritenuta corretta nelle circostanze, ma non per diritto. Similmente nel caso in cui la riparazione o la manutenzione o l'acquisizione di un immobile o edificio fosse di 
interesse generale, la Commissione dovrebbe avere il potere di far fronte a una quota della spesa.

2. Si dovrebbe predisporre l'attività preliminare affinché si elaborino e si rendano operativi dei programmi per non dover aspettare il prossimo crollo, allo scopo di bonificare e ricostruire a spese pubbliche i quartieri insalubri che deturpano le nostre principali città. Prendendo ad esempio Londra, dovremmo demolire la maggior parte degli edifici esistenti nella costa meridionale del fiume dal Palazzo della Contea a Greenwich, e strutturare queste zone come i quartieri operai piu sfarzosi, piu comodi e salubri del mondo. Lo spazio è attualmente così male utilizzato che una popolazione uguale o piu numerosa potrebbe essere ospitata con moderna accoglienza su metà o meno dell'area, destinandone il resto a parchi, piazze e campi da gioco, con laghi, giardini, boulevards, e ogni altro piacere che l'esperienza e la fantasia possono immaginare.

Perchè non dovrebbe l'intera Londra somigliare a St. James's Park e ai suoi dintorni? La riva del fiume potrebbe diventare una delle vedute piu importanti del mondo, con una serie di terrazze ed edifici che sorgono dal fiume. Le scuole della Londra meridionale dovrebbero avere la dignità di università con cortili, colonnati, fontane, librerie, gallerie, saloni da pranzo, cinema e teatri per i propri occupanti. In questo programma si dovrebbe inserire la massima varietà. Tutti i nostri architetti, ingegneri e artisti dovrebbero avere l'opportunità di rappresentare la multiforme immaginazione non di esseri stizzosi, ottusi e disillusi, ma di spiriti pacifici e soddisfatti che appartengono a un rinascimento.

Io sostengo che non c'e nessun ostacolo "finanziario" a tali imprese, dal momento che il lavoro e le risorse materiali sono disponibili. Non è di per se consigliabile mirare alla velocità: i migliori edifici sono pianificati e innalzati lentamente, sono sottoposti a critica paziente e si evolvono sotto gli occhi dell'architetto».

$* * *$

Tuttavia qualsiasi piano di intervento pubblico nel campo dell'arte dovrebbe tenere conto di tre caratteristiche dell'opera d'arte nel mondo in cui viviamo; due di ordine economico, la terza di ordine culturale e sociale:

1. Il valore, dunque il prezzo, di un'opera d'arte non dipende dal suo costo di produzione, ma dalla sua scarsità. Il prezzo di un'opera d'arte manifesta, generalmente, una elevata variabilità e risulta imprevedibile, poichè segue i capricci e le manie del mercato. 
2. Nel suo processo di produzione, un'opera d'arte è soggetta al "paradosso o malattia dei costi" di W. Baumol': un quartetto d'archi di Mozart nel diciannovesimo secolo richiedeva lo stesso numero di musicisti e di strumenti e lo stesso tempo che nel ventunesimo secolo. Nei settori culturali e artistici vi è una continua caduta di competitivitàprezzo, dovuta alla impossibilità di applicare a queste attività il progresso tecnico e i connessi aumenti di produttività.

3. La terza caratteristica, forse la più importante, ha a che fare con un passo di un altro scritto di J.M. Keynes':

Ricordiamo l'epitaffio che scrisse per la sua tomba quella vecchia donna di servizio:

Non portate il lutto, amici, non piangete per me che finalmente non farò niente, niente per l'eternità.

Questo era il suo paradiso. Come altri che aspirano al tempo libero, la donna di servizio immaginava solo quanto sarebbe stato bello passare il tempo a fare da spettatore. C'erano, infatti, altri due versi nell'epitaffio:

Il paradiso risuonerà di salmi e di dolci musiche ma io non farò la fatica di cantare.

Eppure la vita sarà tollerabile solo per quelli che partecipano al canto: e quanto pochi di noi sanno cantare!

Questa terza caratteristica riguarda il momento della fruizione delle opere d'arte, e qui si dovrebbe tenere conto della capacità della popolazione di goderne appieno. Nella sua forma moderna di oggetto di consumo, l'arte presuppone una cultura appropriata - di cui in Italia mancano le premesse più elementari. Secondo i dati raccolti e elaborati

4 W. Baumol, W. Bowen, Performing Arts, The Economic Dilemma: a Study of Problems common to Theater, Opera, Music, and Dance, New York, Twentieth Century Fund, 1966.

5 J.M. Keynes, Economic Possibilities for our Grandchildren, The Nation and Atheneum, 1930. Ora in J.M. Keynes, Essays in Persuasion, London, Macmillan, 1931. Trad.it. in J.M. Keynes, La fine del Laissez-faire e altri scritti, Torino, Bollati Boringhieri, 1991. 
da Tullio De Mauro, 5 italiani su 100 tra i 14 e i 65 anni non sanno distinguere una lettera da un'altra, una cifra dall'altra: sono analfabeti totali; 38 su 100 lo sanno fare, ma riescono solo a leggere con difficoltà una scritta semplice e a decifrare qualche cifra; 33 superano questa condizione, ma qui si fermano: un testo scritto che riguardi fatti collettivi o di rilievo anche nella vita quotidiana, è oltre la portata delle loro capacità di lettura e scrittura, un grafico con qualche percentuale è un'icona indecifrabile. Tra questi, il 12 per cento dei laureati. Soltanto il 20 per cento della popolazione adulta italiana possiede gli strumenti minimi indispensabili di lettura, scrittura e calcolo necessari per orientarsi nella società contemporanea. 